\title{
7. CENOZOIC RADIOLARIANS AT SITE 462, DEEP SEA DRILLING PROJECT LEG 61, WESTERN TROPICAL PACIFIC ${ }^{1}$
}

\author{
Annika Sanfilippo, M. J. Westberg, and W. R. Riedel, Scripps Institution of Oceanography, La Jolla, California
}

\begin{abstract}
Radiolarians are numerous, and moderately to well preserved, in almost all samples from the Pliocene to middle Eocene at this site. The section is, however, practically useless for normal stratigraphic purposes because of the pervasive reworking of older forms into younger levels, which is estimated to account for 30 to $50 \%$ of the assemblages in some samples.
\end{abstract}

\section{INTRODUCTION}

The sequence of radiolarian assemblages at Site 462 $\left(7^{\circ} 14.25^{\prime} \mathrm{N}, 165^{\circ} 01.83^{\prime} \mathrm{E}\right.$, water depth $\left.5181 \mathrm{~m}\right)$ is so confused by reworking of older forms into younger levels that Patrick De Wever asked us to attempt to sort it out while he concentrated on the spyrids, the artostrobiids, and the Cretaceous samples. Our work was based on samples taken and prepared by him.

\section{OBSERVATIONS}

Our results are shown in Table 1. All the preparations examined contained abundant radiolarians, except those from Core 462-1, in which they are sparse. In the tabulation, the preservation of each assemblage is categorized as poor (P), moderate (M), or good (G). Since dissolution often results in the concentration of spyrids, collosphaerids, and orosphaerids, we have kept note of samples in which relative abundances of these groups were higher than normal.

Collosphaerids appear to be concentrated in the preparations from Hole 462, Sections 4-6, 5-1, 5-2, 11-3, $11-4$, and 12-4.

Spyrids appear to be concentrated in the preparations from Hole 462, Sections 10-2, 17-1 through 18-6, 21-5, 26-1, 26-2, 26-3, 26-4, and 30-2.

Orosphaerids appear to be concentrated in the preparations from Sections 462-12-1 and 462-16-2.

High concentrations of sponge spicules, possibly resulting from selective dissolution and/or from influx of shallower-water material, were noted in the preparations from Hole 462, Sections 16-2, 18-6, 20-3, 20-4, 23-2 through 23-6, Sample 34,CC, Sections 38-2 and 38-4, Sample 38,CC, and Section 39-3.

Entries in the column headed "Sorting?" indicate whether the assemblage seems to be normal $(\mathrm{N})$, abnormally coarse $(\mathrm{C})$, abnormally fine $(\mathrm{F})$, or to have the medium grain sizes concentrated by winnowing (W).

The main body of the tabulation shows estimated relative abundances of species, according to the following scheme: abundant (A), tens of thousands of specimens on a slide; common (C), thousands; few (F),

\footnotetext{
' Initial Reports of the Deep Sea Drilling Project, Volume 61.
}

50-1000; rare (R), 3-50; very rare $(+), 1$ or 2 . On each slide are about 20,000 to 40,000 radiolarians.

The columns headed "Estimated Reworking" give Riedel's estimates (as percentages) of radiolarians of different ages reworked into each assemblage. These numbers are of course very rough. All concentrations up to $1 \%$ are recorded as 1 .

The relationship between radiolarian zones and epoch boundaries is nearly always established by correlations with calcareous microfossils. A basis for establishing this relation is shown in Figure 1, in which the limits of radiolarian zones are shown in relation to calcareous-nannofossil zones and sub-zones of Okada and Bukry (1980). This was done by surveying all of the DSDP Initial Reports volumes from Leg 10 through Leg 50 , and recording the relations of the critical radiolarian events to Bukry's zonal assignments in those volumes.

\section{COMMENTS}

In the tabulation, species are arranged in order of their first occurrences. Because of the extensive reworking in the section, these lowest occurrences constitute the principal basis of our stratigraphic interpretation. As is evident from the left-hand side of the table, the placement of some zonal boundaries is uncertain because of our inablilty to determine evolutionary transitions and upper limits of stratigraphic ranges in this sequence.

There seem also to be some departures from the usual order of earliest appearances (as summarized by Riedel and Sanfilippo, 1978), which should be investigated in nearby sequences less affected by reworking. The most striking of these anomalies are the following:

1) The earliest occurrences of Podocyrtis ampla ampla and $P$. ampla fasciolata are abnormally high.

2) The earliest occurrences of Thyrsocyrtis bromia and Calocyclas turris are abnormally low in relation to the first occurrences of Theocampe pirum, the Lithocyclia aristotelis group, Thyrsocyrtis tetracantha, and Carpocanistrum azyx.

3) The earliest occurrences of Stichocorys delmontensis and Artostrobium doliolum are abnormally low.

4) The isolated, abnormally low occurrences of Siphocampe corbula, Dictyocoryne ontongensis, and Spongaster tetras in Sample 462-15,CC can be explained only by their having fallen down the hole. 


\begin{tabular}{|c|c|c|c|c|c|}
\hline $\begin{array}{l}\text { Martini } \\
\text { (1971) } \\
\text { Zones }\end{array}$ & \multicolumn{4}{|c|}{$\begin{array}{l}\text { Okada and Bukry (in press) Calcareous-Nannofossil } \\
\text { Zones }\end{array}$} & $\begin{array}{l}\text { Radiolarian Zones, Events, and } \\
\text { Ages (Theyer et al., 1978) }\end{array}$ \\
\hline NN21 & CN15 & \multicolumn{3}{|c|}{ Emiliania huxleyi } & \multirow{3}{*}{ Lamprocyrtis haysi Zone } \\
\hline NN20 & \multirow{2}{*}{ CN14 } & Geophyrocapsa & CN14b & C. cristatus & \\
\hline \multirow{3}{*}{ NN19 } & & oceanica & CN14a & E. ovata & \\
\hline & \multirow{2}{*}{$\mathrm{CN} 13$} & \multirow{2}{*}{$\begin{array}{c}\text { Crenalithus } \\
\text { doronicoides }\end{array}$} & CN13b & G. caribbeanica & \multirow{4}{*}{$\begin{array}{l}\text { - L. neoheteroporos } \rightarrow \text { L. haysi }- \\
\text { Pterocanium prismatium Zone }\end{array}$} \\
\hline & & & CN13a & E. annula & \\
\hline NN18 & \multirow{4}{*}{$\mathrm{CN} 12$} & \multirow{4}{*}{$\begin{array}{l}\text { Discoaster } \\
\text { brouweri }\end{array}$} & CN12d & C. macintyrei & \\
\hline NN17 & & & CN12c & D. pentaradiatus & \\
\hline NN16 & & & CN12b & D. sulculus & — Tm S. peregrina ( 2.5 m. y.) \\
\hline \multirow{3}{*}{ NN15 } & & & CN12a & D. tamalis & \multirow{3}{*}{ Spongaster pentas Zone } \\
\hline & \multirow{2}{*}{ CN11 } & \multirow{2}{*}{$\begin{array}{l}\text { Reticulofenestra } \\
\text { pseudoumbilica }\end{array}$} & CN11b & D. asymmetricus & \\
\hline & & & CN11a & S. neoabies & \\
\hline $13 / 14$ & \multirow[b]{2}{*}{ CN10 } & \multirow{2}{*}{$\begin{array}{l}\text { Amaurolithus } \\
\text { tricorniculatus }\end{array}$} & CN10c & C. rugosus & \multirow{3}{*}{$\begin{array}{c}\text { - S. berminghami } \rightarrow \text { S. pentas }(4.4 \mathrm{~m} . \text { y. })- \\
\text { Stichocorvs peregrina Zone }\end{array}$} \\
\hline NN12 & & & CN10b & C. acutus & \\
\hline & \multirow[b]{2}{*}{ CN9 } & \multirow[b]{2}{*}{$\begin{array}{l}\text { Discoaster } \\
\text { quinqueramus }\end{array}$} & $\begin{array}{ll}\text { CN10a } \\
\text { CN9b }\end{array}$ & $\frac{\text { T. rugosus }}{\text { A. primus }}$ & \\
\hline NN11 & & & CN9a & D. berggrenii & $\begin{array}{c}\text { - S. delmontensis } \rightarrow \text { S. peregrina }(6.2 \mathrm{~m} . \text { y.) } \\
\text { Ommatartus penultimus Zone } \\
-\mathrm{Tm} \text { O. hughesi }(7.8 \mathrm{~m} . \text { y.) }\end{array}$ \\
\hline & & Discoaster & CN 8b & D. neorectus & Ommatartus antepenultimus Zone \\
\hline NN10 & CN8 & neohamatus & CN8a & D. bellus & $C$ netterseani $\rightarrow O$ huahes \\
\hline NN9 & CNד & Discoaster & CN7b & C. calyculus & C. pettersson $\rightarrow$ U. nughes \\
\hline NN9 & CN7 & hamatus & CN7a & H. carteri & \\
\hline NN8 & CN6 & Catinaster coalitus & & & Cannartus petterssoni Zone \\
\hline NN7 & & Discoaster & CN5b & D. kugleri & Bm C. petterssoni $(11.2 \mathrm{~m} . \mathrm{y}) \longrightarrow$ \\
\hline NN6 & CN5 & exilis & CN5a & C. miopelagicus & $\begin{array}{l}\text { Dorcadospyris alata Zone } \\
\text { Dota }\end{array}$ \\
\hline NN5 & $\mathrm{CN} 4$ & Sphenolithus heteromorph & & & - D. dentata $\longrightarrow$ D. alata (15.4 m. y.) - \\
\hline & CN3 & Helicosphaera ampliaperta & & & Calocycletta costata Zone \\
\hline NN2 & $\mathrm{CN} 2$ & Sphenolithus belemos & & & $\begin{array}{l}\text { Sm C. costata (18.4 m. y.) } \\
\text { Stichocorys wolffii Zone }\end{array}$ \\
\hline & & & $\mathrm{CN} 1 \mathrm{C}$ & D. druggii & $\begin{array}{l}\text { - Bm S. wolffii (19.2 m. y.) } \\
\text { Stichocorys delmontensis Zone }\end{array}$ \\
\hline NN1 & CN1 & Triquetrorhabdulus & CN1b & D. deflandrei & $\begin{array}{l}\text { - Tm T. annosa }(22.2 \mathrm{~m} . \text { y.) } \\
\text { Cyrtocapsel/a tetrapera Zone }\end{array}$ \\
\hline & & & & & - Bm C. tetrapera (22.6 m. y.) \\
\hline & & & CN1a & C. abisectus & Lychnocanoma elongata Zone \\
\hline NP25 & & Sphenolithus & CP19b & D. bisectus & Bm L. elongata (24.2 m. y.) \\
\hline NP24 & CP19 & ciperoensis & CP19a & C. tloridanus & Dorcadospyris ateuchus Zone \\
\hline NP23 & CP18 & Sphenolithus distentus & & & $-T$. triceros $\rightarrow D$. ateuchus \\
\hline NP23 & $\mathrm{CP} 17$ & Sphenolithus predistentus & & & \\
\hline NP22 & & & CP16c & R. hillae & Theocyrtis tuberosa Zone \\
\hline NP21 & CP16 & $\begin{array}{l}\text { Helicospnaera } \\
\text { reticulata }\end{array}$ & CP16b & C. formosus & $-L$. aristotelis $\rightarrow L$. angusta \\
\hline & & & CP16a & C. subdistichus & \\
\hline $19 / 20$ & $C D 15$ & Discoaster & CP15b & 1. recurvus & Thyrsocyrtis bromia Zone \\
\hline NP18 & & barbadiensis & CP15a & C. oamaruensis & \\
\hline NP17 & & Reticulofenestra & $\mathrm{CP} 14 \mathrm{~b}$ & D. saipanensis & $\begin{array}{l}\text { - Bm C. azyx } \\
\text { Podocyrtis goetheana Zone } \\
\text { Bm P. goetheana }\end{array}$ \\
\hline & CP14 & umbilica & & & Podocyrtis chalara Zone \\
\hline ND16 & & & $\mathrm{CP} 14 \mathrm{a}$ & D. bifax & Podocyrtis mitra Zone \\
\hline NP16 & & & $\mathrm{CP} 13 \mathrm{c}$ & C. staurion & $\begin{array}{l}-P . \text { sinuosa } \rightarrow \text { P. mitra } \\
\text { Podocyrtis ampla Zone }\end{array}$ \\
\hline & CP13 & Nannotetrina & CP13b & C. gigas & P. phyxis $\rightarrow$ P. ampla \\
\hline NP15 & & & CP13a & D. strictus & Thyrsocyrtis triantha Zone \\
\hline & & & $\mathrm{CP} 12 \mathrm{~b}$ & R. inflata & - Bm E. lagena Theocampe mongolfieri Zone \\
\hline NP14 & CP12 & Discoaster & & & $\begin{array}{l}\text { - Bm T. mongolfieri } \\
\text { Theocotyle cryptocephala Zone }\end{array}$ \\
\hline & & & $\mathrm{CP} 12 \mathrm{a}$ & D. kuepperi & - T. nigriniae $\rightarrow T$. cryptocephala \\
\hline & CP11 & Discoaster lodoensis & & & $\begin{array}{l}\text { Phormocyrtis striata Zone } \\
\text { - }\end{array}$ \\
\hline $12 / 13$ & CP10 & Tribrachiatus orthostylus & & & Bm T. anaclasta $\quad$ Burvella clinata Zone \\
\hline NP11 & & Discoaster & $\mathrm{CP9b}$ & D. binodosus & B. tetradica $\rightarrow$ B. clinata \\
\hline NP10 & CP9 & diastypus & CP9a & T. contortus & \\
\hline
\end{tabular}

Figure 1. Relation of radiolarian zones to calcareous-nannofossil zones. Numbers in parentheses indicate ages in millions of years. 
Parts of the sequence in Hole 462 that seem not to be seriously disturbed by reworking are the major part of Core 5, the lower half of Core 6, Core 8 through the upper part of Core 12, the lower half of Core 13 through Core 18, the lower half of Core 19, the lower half of Core 21 , Cores 24 through 25, most of Core 27, the lower part of Core 28, the lower part of Core 29 through Core 31, the bottom of Core 32 and the top of Core 33, and Cores 35 through 36 . In Hole $462 \mathrm{~A}$, Core 1 seems to be not much disturbed by reworking. These parts of the sequence are the most useful for locating biostratigraphic boundaries.

There is some discrepancy between the correlation of the top two cores in Hole $462 \mathrm{~A}$ with the sequence in Hole 462 , as indicated by the radiolarians, and that which would be expected on the basis of drilling depth. The radiolarians in Core $462 \mathrm{~A}-1$ are similar to those above the bottom of Core 462-8, and the assemblages in Core $462 \mathrm{~A}-2$ are similar to those in Cores $462-22$ to 462-24.

\section{SPECIES LIST}

The purpose of this list is to provide bibliographic references to the taxa mentioned in this chapter. The only literature references given are to the original description, and to our present concept of the species if different from or more detailed than the original one.

Acrobotrys tritubus Riedel

Acrobotrys tritubus Riedel, 1957, p. 80, pl. 1, fig. 5. Riedel and Sanfilippo, 1978 , p. 65 , pl. 3 , fig. 1 .

Artophormis gracilis Riedel

Artophormis gracilis Riedel, 1959, p. 300, pl. 2, figs, 12, 13.

Artostrobium doliolum Riedel and Sanfilippo

Artostrobium doliolum Riedel and Sanfilippo, 1971, p. 1599, pl. $1 \mathrm{H}$, figs. $1-3$; pl. 8 , figs. 14,15 . Westberg and Riedel, 1978, p. 20 , pl. 3, figs. 10, 11 .

Buryella clinata Foreman

Buryella clinata Foreman, 1973, p. 433, pl. 8, figs. 1-3; pl. 9, fig.

19. Riedel and Sanfilippo, 1978, p. 65, pl. 3, fig. 4.

Calocyclas hispida (Ehrenberg)

Anthocyrtis hispida Ehrenberg, 1873, p. 216.

Calocyclas hispida (Ehrenberg), Riedel and Sanfilippo, 1978, p. 65 , pl. 3 , fig. 6.

Calocyclas turris Ehrenberg

Calocyclas turris Ehrenberg, 1873, p. 218. Riedel and Sanfilippo, 1978 , p. 65 , pl. 3 , figs. 7,8 .

Calocycletta costata (Riedel) Calocyclas costata Riedel, 1959, p. 296, pl. 2, fig. 9. Riedel and Sanfilippo, 1978, p. 66 , pl. 3, fig. 9.

Calocycletta virginis Haeckel

Calocyclas (Calocycletta) virginis Haeckel, 1887, p. 1381, pl. 74, fig. 4.

Calocycletta virginis (Haeckel), Riedel and Sanfilippo, 1978, p. 66, pl. 3, figs. 13, 14.

Cannartus laticonus Riedel

Cannartus laticonus Riedel, 1959, p. 291, pl. 1, fig. 5. Westberg and Riedel, 1978, p. 20, pl. 2, figs. 1-3.

Cannartus petterssoni Riedel and Sanfilippo Cannartus(?) petterssoni Riedel and Sanfilippo, 1970, p. 520, pl. 14, fig. 3. Riedel and Sanfilippo, 1978, p. 67, pl. 4, fig. 2.

Carpocanistrum azyx Sanfilippo and Riedel Carpocanistrum azyx Sanfilippo and Riedel, 1973, p. 530, pl. 35, fig. 9. Riedel and Sanfilippo, 1978, p. 67, pl. 4, fig. 5.

Carpocanopsis bramlettei Riedel and Sanfilippo

Carpocanopsis bramlettei Riedel and Sanfilippo, 1971, p. 1597, pl. 2G, figs. 8-14; pl. 8, fig. 7. Riedel and Sanfilippo, 1978, p. 67, pl. 4 , fig. 6.

Carpocanopsis cingulata Riedel and Sanfilippo

Carpocanopsis cingulata Riedel and Sanfilippo, 1971, p. 1597, pl.
2G, figs. 17-21; pl. 8, fig. 8. Riedel and Sanfilippo, 1978, p. 67, pl. 4 , fig. 4.

Centrobotrys gravida Moore

Centrobotrys gravida Moore, 1971, p. 744, pl. 5, fig. 8. Riedel and Sanfilippo, 1978, p. 67, pl. 4, fig. 8.

Cyclampterium milowi Riedel and Sanfilippo

Cyclampterium milowi Riedel and Sanfilippo, 1971, p. 1593, pl. 3B, fig. 3; pl. 7, figs. 8, 9. Riedel and Sanfilippo, 1978, p. 67, pl. 4, fig. 14.

Cyrtocapsella cornuta Haeckel

Cyrtocapsa (Cyrtocapsella) cornuta Haeckel, 1887, p. 1513, pl. 78, fig. 9.

Cyrtocapsella cornuta Haeckel, Riedel and Sanfilippo, 1978, p. 68, pl. 4, fig. 17.

Cyrtocapsella tetrapera Haeckel

Cyrtocapsa (Cyrtocapsella) tetrapera Haeckel, 1887, p. 1512, pl. 78 , fig. 5 .

Cyrtocapsella tetrapera Haeckel, Riedel and Sanfilippo, 1978, p. 68, pl. 4, fig. 18.

Dictyocoryne ontongensis Riedel and Sanfilippo

Dictyocoryne ontongensis Riedel and Sanfilippo, 1971, p. 1588, pl. 1E, figs. 1, 2; pl. 4, figs. 9-11. Riedel and Sanfilippo, 1978, p. 68 , pl. 5, fig. 1 .

Dictyophimus craticula Ehrenberg

Dictyophimus craticula Ehrenberg 1873, p. 223. Riedel and Sanfilippo, 1978, p. 68, pl. 4, fig. 19.

Dorcadospyris alata (Riedel)

Brachiospyris alata Riedel, 1959, p. 293, pl. 1, figs. 11, 12

Dorcadospyris alata (Riedel), Riedel and Sanfilippo, 1978, p. 68 , pl. 5, fig. 2.

Dorcadospyris ateuchus (Ehrenberg)

Ceratospyris ateuchus Ehrenberg, 1873, p. 218.

Dorcadospyris ateuchus (Ehrenberg), Riedel and Sanfilippo. 1978, p. 68 , pl. 5 , fig. 3 .

Dorcadospyris dentata Haeckel

Dorcadospyris dentata Haeckel, 1887, p. 1040, pl. 85, fig. 6 . Riedel and Sanfilippo, 1978, p. 68, pl. 5, fig. 4.

Eusyringium fistuligerum (Ehrenberg)

Eucyrtidium fistuligerum Ehrenberg, 1873, p. 229.

Eusyringium fistuligerum (Ehrenberg), Riedel and Sanfilippo, 1978 , p. 68 , pl. 5, figs. $6,7$.

Eusyringium lagena (Ehrenberg)

Lithopera lagena Ehrenberg, 1873, p. 241.

Eusyringium lagena (Ehrenberg), Riedel and Sanfilippo, 1978, p. 68 , pl. 5 , fig. 8 .

Lamptonium fabaeforme chaunothorax Riedel and Sanfilippo Lamptonium fabaeforme chaunothorax Riedel and Sanfilippo, 1970 , p. 524, pl. 5, figs. 8, 9. Riedel and Sanfilippo, 1978, p. 69, pl. 5 , fig. 11 .

Lamptonium fabaeforme constrictum Riedel and Sanfilippo Lamptonium fabaeforme constrictum Riedel and Sanfilippo, 1970 , p. 523, pl. 5, fig. 7. Riedel and Sanfilippo, 1978, p. 69, pl. 5, fig. 12.

Lithochytris vespertilio Ehrenberg

Lithochytris vespertilio Ehrenberg, 1873, p. 239. Riedel and Sanfilippo, 1978 , p. 69 , pl. 6 , fig. 4 .

Lithocyclia angusta (Riedel)

Trigonactura angusta Riedel, 1959, p. 292, pl. 1, fig. 6.

Lithocyclia angusta (Riedel), Riedel and Sanfilippo, 1978, p. 70, pl. 6 , fig. 5 .

Lithocyclia aristotelis (Ehrenberg) group Astromma aristotelis Ehrenberg, 1847, p. 55, fig. 10. Lithocyclia aristotelis (Ehrenberg) group, Riedel and Sanfilippo, 1978, p. 70 , pl. 6 , fig. 6 .

Lithocyclia crux Moore Lithocyclia crux Moore, 1971, p. 737, pl. 6, fig. 4. Riedel and Sanfilippo, 1978, p. 70, pl. 6, fig. 7 .

Lithocyclia ocellus Ehrenberg group Lithocyclia ocellus Ehrenberg, 1854, pl. 36, fig. 30. Lithocyclia ocellus Ehrenberg group, Riedel and Sanfilippo, 1978, p. 70 , pl. 6 , fig. 8 .

Lithopera neotera Sanfilippo and Riedel Lithopera neotera Sanfilippo and Riedel, 1970, p. 454, pl. 1, figs. 24-26, 28. Riedel and Sanfilippo, 1978, p. 70, pl. 6, fig. 10. 
A. SANFILIPPO, M. J. WESTBERG, W. R. RIEDEL

Table 1. Occurrences of radiolarian species in samples from Site 462. (Abbreviations explained in text.)

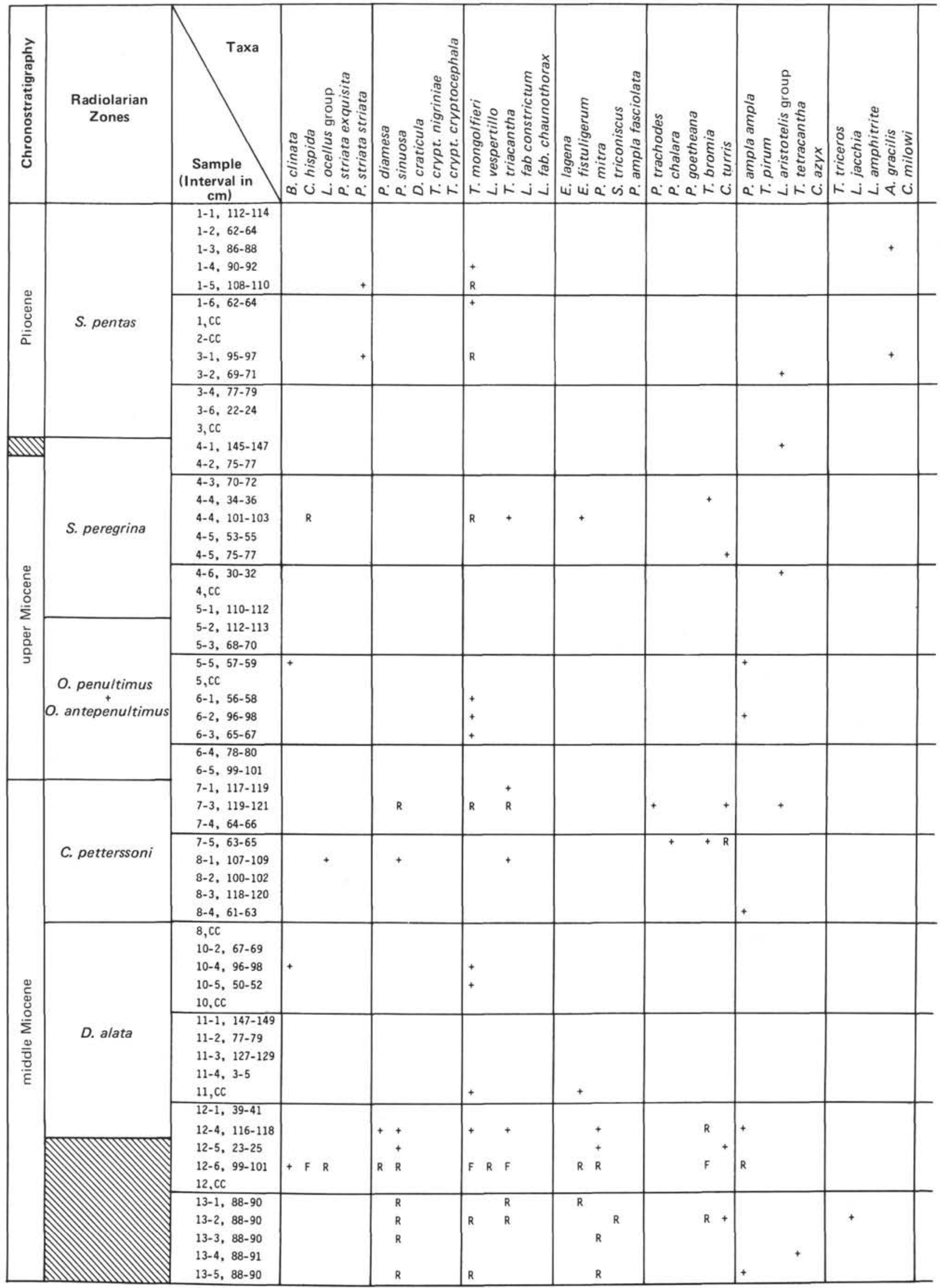


Table 1. (Continued).

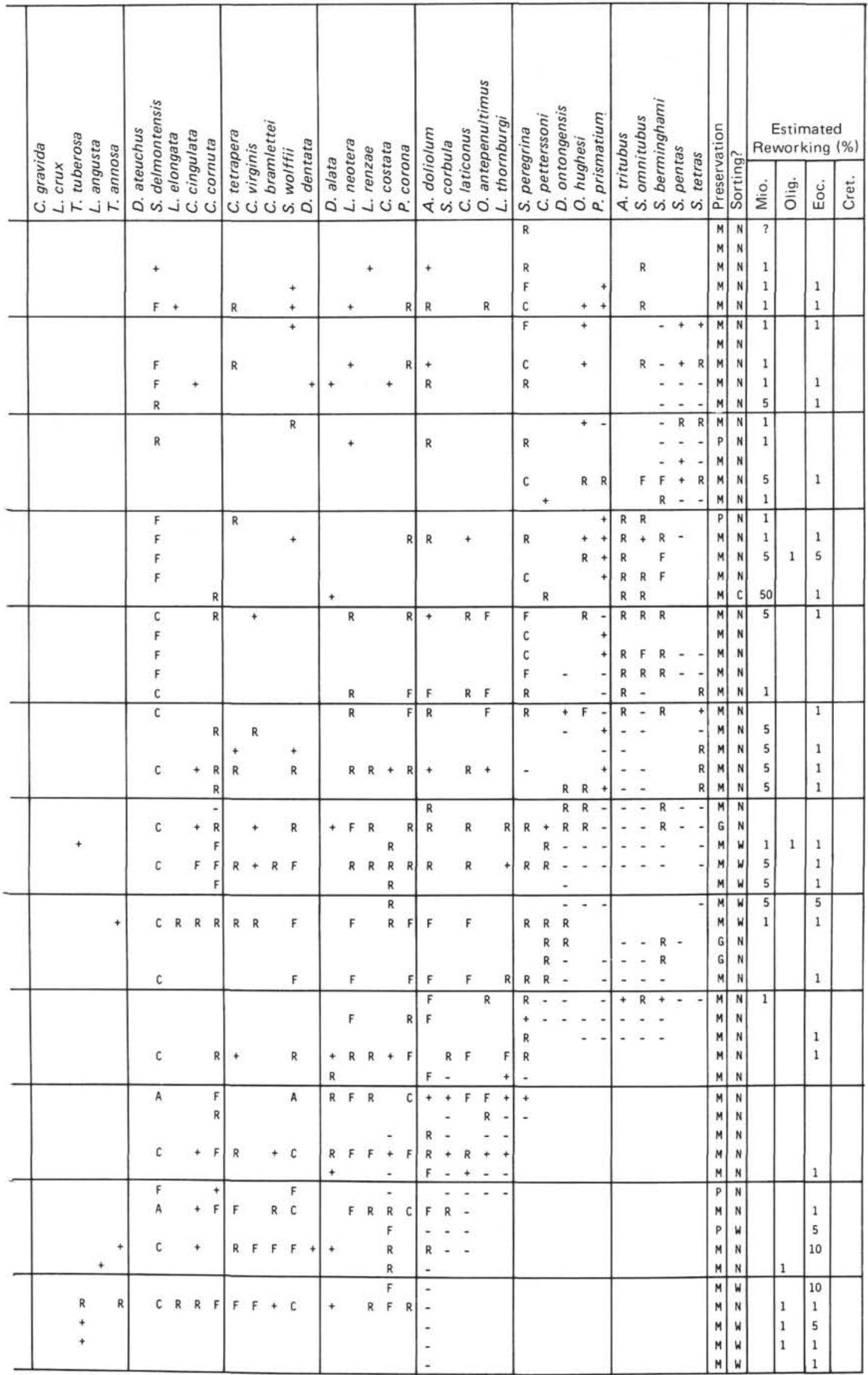


Table 1. (Continued).

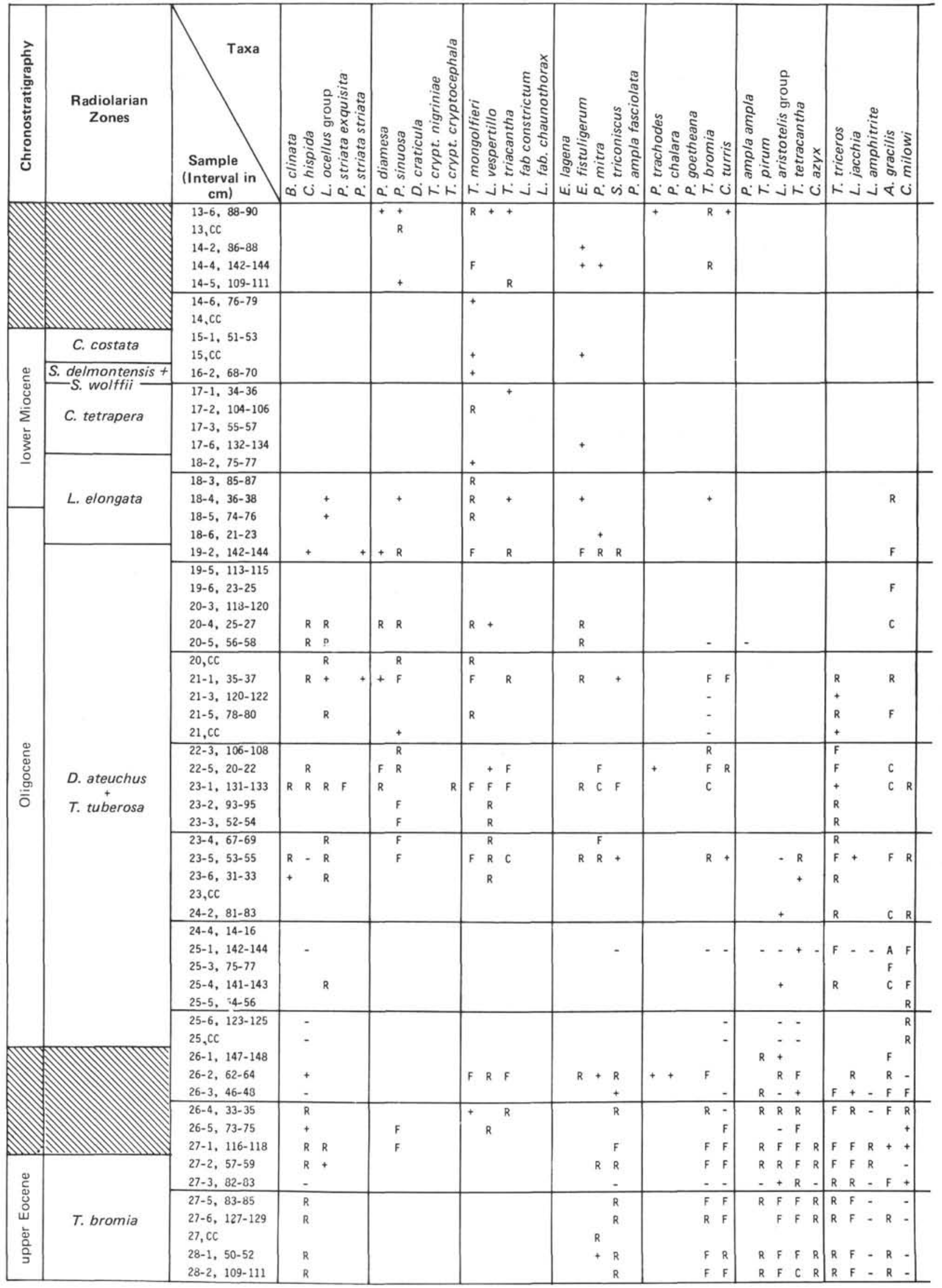


Table 1. (Continued).

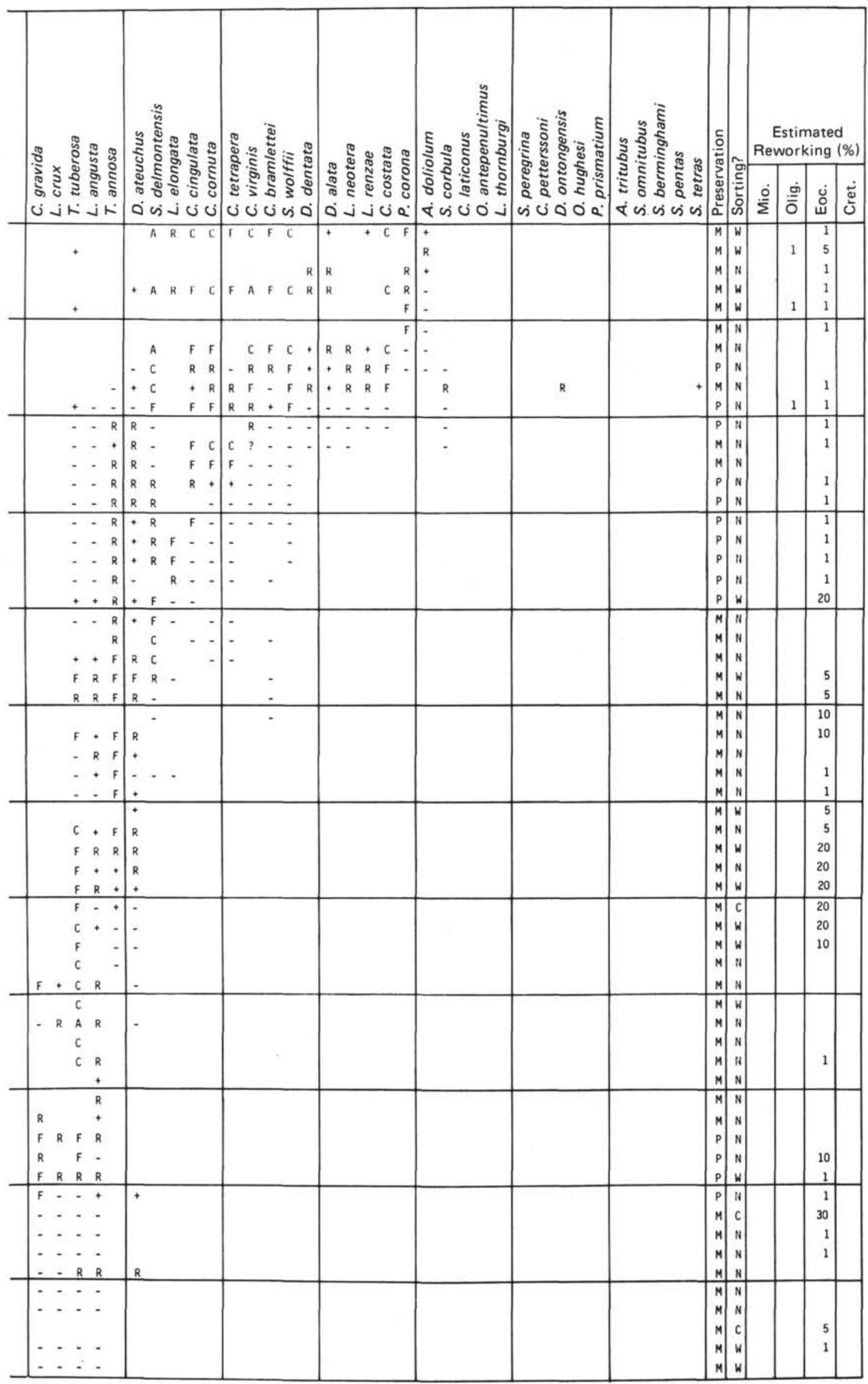


Table 1. (Continued).

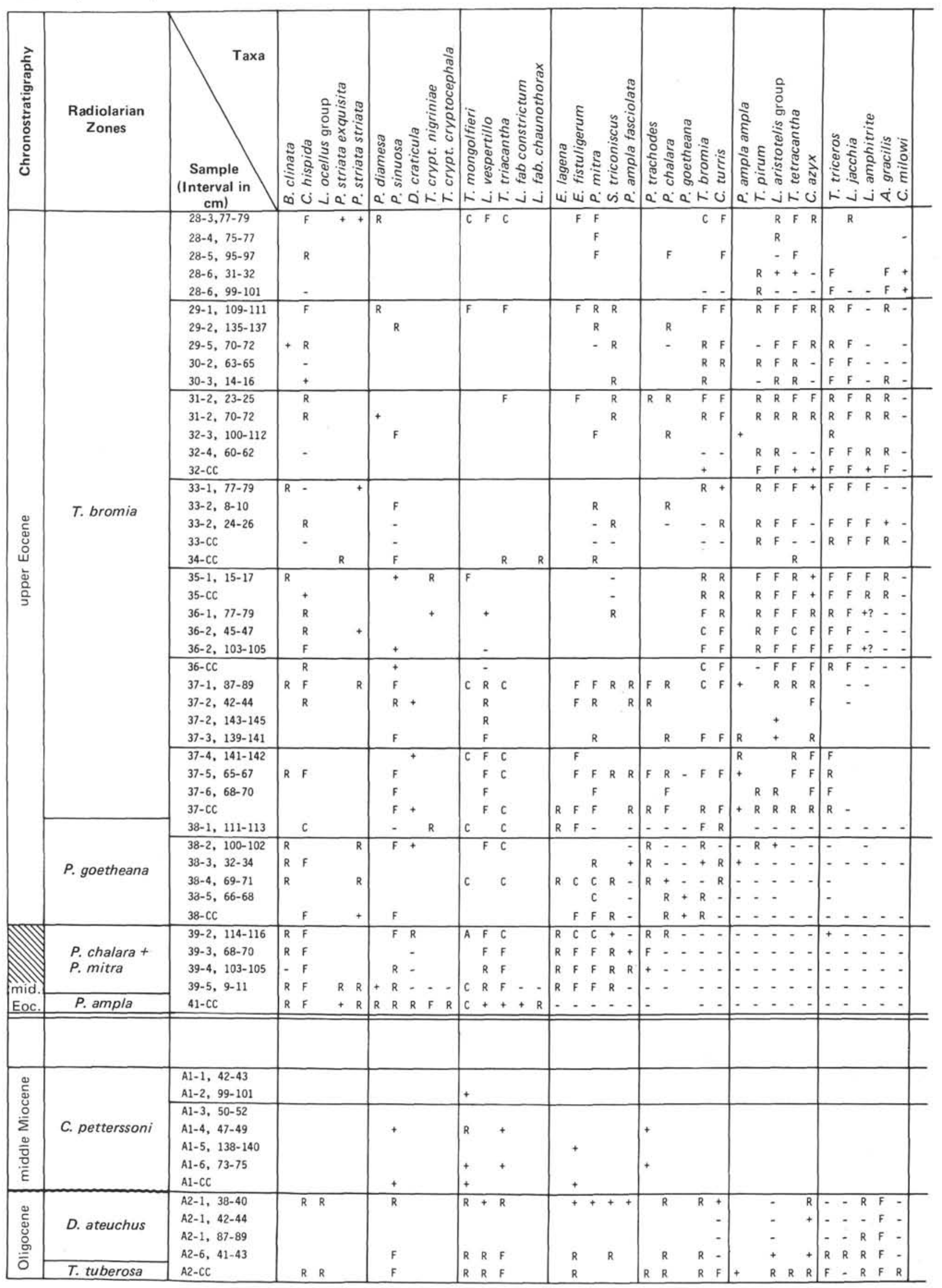


Table 1. (Continued).

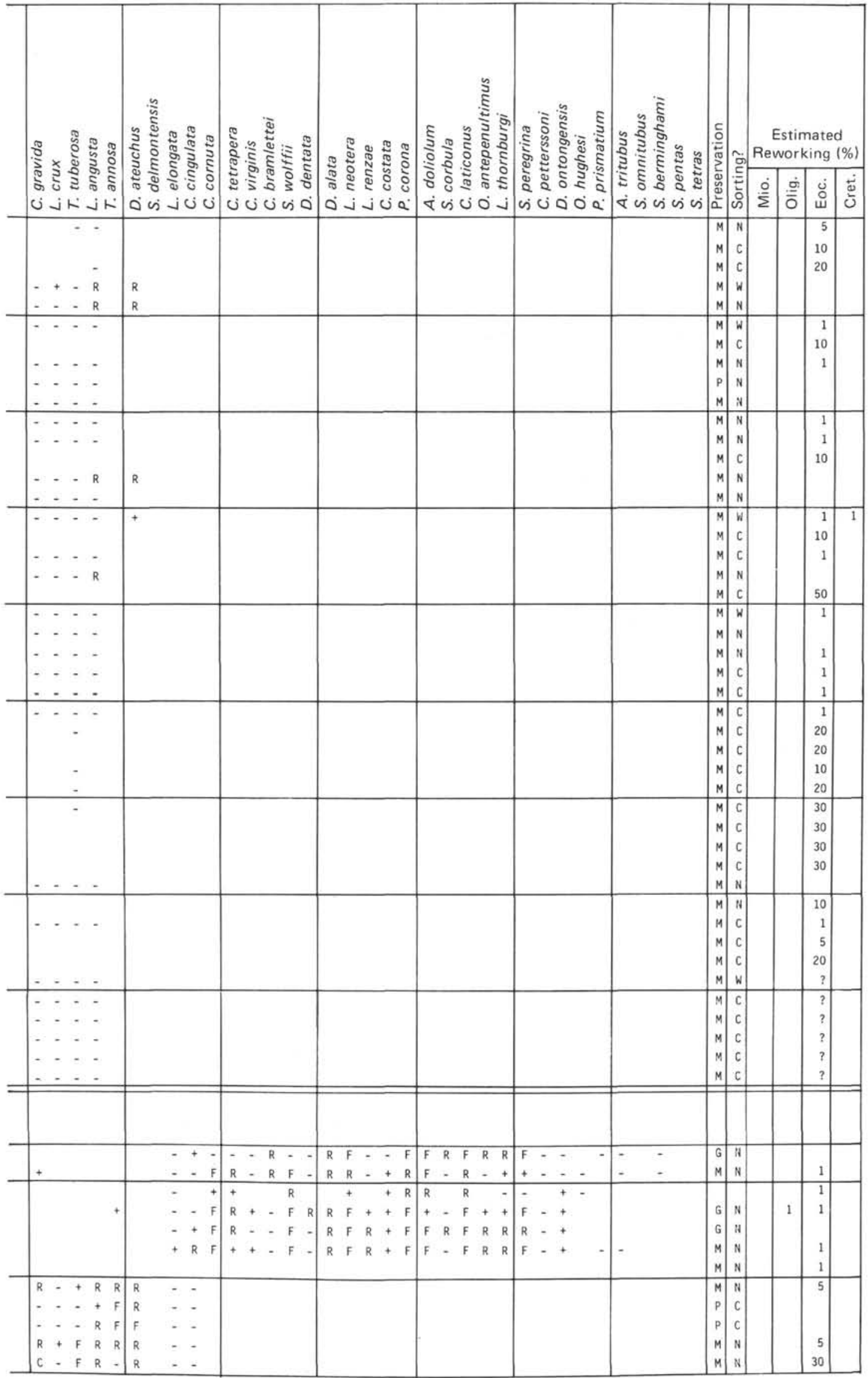


Lithopera renzae Sanfilippo and Riedel

Lithopera renzae Sanfilippo and Riedel, 1970, p. 454, pl. 1, figs. 21-23, 27. Riedel and Sanfilippo, 1978, p. 70, pl. 6, fig. 11.

Lithopera thornburgi Sanfilippo and Riedel

Lithopera thornburgi Sanfilippo and Riedel, , 1970, p. 455, pl. 2, figs. 4-6. Riedel and Sanfilippo, 1978, p. 70, pl. 6, fig. 12.

Lophocyrtis jacchia (Ehrenberg)

Thyrsocyrtis jacchia Ehrenberg, 1873, p. 261.

Lophocyrtis jacchia (Ehrenberg), Riedel and Sanfilippo, 1978, p. 70 , pl. 7, fig. 1.

Lychnocanoma amphitrite Foreman

Lychnocanoma amphitrite Foreman, 1973, p. 437, pl. 11, fig. 10. Riedel and Sanfilippo, 1978, p. 70, pl. 7, figs. 2, 3.

Lychnocanoma elongata (Vinassa)

Tetrahedrina elongata Vinassa, 1900, p. 243, pl. 2, fig. 31.

Lychnocanoma elongata (Vinassa), Riedel and Sanfilippo, 1978, p. 70 , pl. 7 , fig. 4 .

Ommatartus antepenultimus Riedel and Sanfilippo

Ommatartus antepenultimus Riedel and Sanfilippo, 1970, p. 521, pl. 14, fig. 4. Westberg and Riedel, 1978, p. 22, pl. 2, figs. 4, 5.

Ommatartus hughesi (Campbell and Clark)

Ommatocampe hughesi Campbell and Clark, 1944, p. 23, pl. 3, fig. 12.

Ommatartus hughesi (Campbell and Clark), Riedel and Sanfilippo, 1978, p. 71, pl. 7, fig. 7.

Phormocyrtis striata exquisita (Kozlova)

Podocyrtis exquisita Kozlova, in Kozlova and Gorbovetz, 1966, p. 106, pl. 17, fig. 2.

Phormocyrtis striata exquisita (Kozlova), Riedel and Sanfilippo, 1978, p. 71, pl. 7, fig. 10.

Phormocyrtis striata striata Brandt

Phormocyrtis striata Brandt, 1935, in Wetzel, 1935, p. 55, pl. 9, fig. 12.

Phormocyrtis striata striata Brandt, Riedel and Sanfilippo, 1978, p. 71 , pl. 7, fig. 11 .

Phormostichoartus corona Haeckel

Cyrtophormis (Acanthocyrtis) corona Haeckel, 1887, p. 1462, pl. 77, fig. 15.

Phormostichoartus corona Haeckel, Riedel and Sanfilippo, 1978, p. 71, pl. 7 , fig. 12 .

Podocyrtis ampla ampla Ehrenberg

Podocyrtis ampla Ehrenberg, 1873, p. 248. Riedel and Sanfilippo, 1978 , p. 71 , pl. 8 , fig. 1.

Podocyrtis ampla fasciolata Nigrini

Podocyrtis ampla fasciolata Nigrini, 1974, p. 1069, pl. 1K, figs. 1, 2; pl. 4, figs. 2, 3. Riedel and Sanfilippo, 1978, p. 71, pl. 8, fig. 2.

Podocyrtis chalara Riedel and Sanfilippo

Podocyrtis chalara Riedel and Sanfilippo, 1970, p. 535, pl. 12, figs. 2, 3. Riedel and Sanfilippo, 1978, p. 71, pl. 8, fig. 3.

Podocyrtis diamesa Riedel and Sanfilippo

Podocyrtis diamesa Riedel and Sanfilippo, 1970, p. 533, pl. 12, figs. 4-6. Riedel and Sanfilippo, 1978, p. 72, pl. 8, fig. 4.

Podocyrtis goetheana (Haeckel)

Cycladophora goetheana Haeckel, 1887, p. 1376, pl. 65, fig. 5. Podocyrtis goetheana (Haeckel), Riedel and Sanfilippo, 1978, p. 72 , pl. 8, fig. 6 .

Podocyrtis mitra Ehrenberg

Podocyrtis mitra Ehrenberg, 1854, pl. 36, fig. B20. Riedel and Sanfilippo, 1978, p. 72 , pl. 8, fig. 7 .

Podocyrtis sinuosa Ehrenberg

Podocyrtis sinuosa Ehrenberg, 1873, p. 253. Riedel and Sanfilippo, 1978 , p. 72 , pl. 8 , fig. 9 .

Podocyrtis trachodes Riedel and Sanfilippo

Podocyrtis trachodes Riedel and Sanfilippo, 1970, p. 535, pl. 11, fig. 7; pl. 12, fig. 1. Riedel and Sanfilippo, 1978, p. 72, pl. 8, fig. 10.

Pterocanium prismatium Riedel

Pterocanium prismatium Riedel, 1957, p. 87, pl. 3, figs. 4, 5. Riedel and Sanfilippo, 1978, p. 72, pl. 9, fig. 1 .

Sethochytris triconiscus Haeckel

Sethochytris triconiscus Haeckel, 1887, p. 1239, pl. 57, fig. 13. Riedel and Sanfilippo, 1978, p. 73, pl. 9, fig. 6.

Siphocampe corbula (Harting)

Lithocampe corbula Harting, 1863, p. 12, pl. 1, fig. 21.
Siphocampe corbula (Harting), Riedel and Sanfilippo, 1978, p. 73, pl. 9 , fig. 7 .

Solenosphaera omnitubus Riedel and Sanfilippo

Solenosphaera omnitubus omnitubus Riedel and Sanfilippo, 1971, p. 1586, pl. 1A, fig. 24; pl. 4, figs. 1, 2. Riedel and Sanfilippo, 1978 , p. 73 , figs. $8,9$.

Spongaster berminghami (Campbell and Clark)

Spongasteriscus berminghami Campbell and Clark, 1944, p. 30, pl. 5, figs. 1, 2.

Spongaster berminghami (Campbell and Clark), Riedel and Sanfilippo, 1978, p. 73, pl. 2, figs. 14-16.

Spongaster pentas Riedel and Sanfilippo

Spongaster pentas Riedel and Sanfilippo, 1970, p. 523, pl. 15, fig. 3. Riedel and Sanfilippo, 1978, p. 74, pl. 2, figs. 5-8.

Spongaster tetras Ehrenberg

Spongaster tetras Ehrenberg, 1860, p. 833. Riedel and Sanfilippo, 1978 , p. 74 , pl. 2 , figs. 2 , 3 .

Stichocorys delmontensis (Campbell and Clark)

Eucyrtidium delmontense Campbell and Clark, 1944, p. 56, pl. 7, figs. 19, 20.

Stichocorys delmontensis (Campbell and Clark), Westberg and Riedel, 1978, p. 22, pl. 3, figs. 1-5.

Stichocorys peregrina (Riedel)

Eucyrtidium elongatum peregrinum Riedel, 1953, p. 812, pl. 85, fig. 2.

Stichocorys peregrina (Riedel), Westberg and Riedel, 1978, p. 22, pl. 3, figs. 6-9.

Stichocorys wolffii Haeckel

Stichocorys wolffii Haeckel, 1887, p. 1479, pl. 80, fig, 10. Riedel and Sanfilippo, 1978, p. 74, pl. 9, fig. 12.

Theocampe mongolfieri (Ehrenberg)

Eucyrtidium mongolfieri Ehrenberg, 1854, pl. 36, fig. 18B.

Theocampe mongolfieri (Ehrenberg), Riedel and Sanfilippo, 1978, p. 76, pl. 9, fig. 13 .

Theocampe pirum (Ehrenberg)

Eucyrtidium pirum Ehrenberg, 1873, p. 232.

Theocampe pirum (Ehrenberg), Riedel and Sanfilippo, 1978, p. 76 , pl. 9, fig. 14.

Theocotyle cryptocephala cryptocephala (Ehrenberg)

Eucyrtidium cryptocephalum Ehrenberg, 1873, p. 227.

Theocotyle cryptocephala cryptocephala (Ehrenberg), Riedel and Sanfilippo, 1978, p. 78, pl. 9, fig. 19.

Theocotyle cryptocephala nigriniae Riedel and Sanfilippo Theocotyle cryptocephala nigriniae Riedel and Sanfilippo, 1970, p. 525, pl. 6, figs. 5, 6. Riedel and Sanfilippo, 1978, p. 78, pl. 9, fig. 19.

Theocyrtis annosa (Riedel)

Phormocyrtis annosa Riedel, 1959, p. 295, pl. 2, fig. 7.

Theocyrtis annosa (Riedel), Riedel and Sanfilippo, 1978, p. 78, pl. 10 , fig. 3.

Theocyrtis tuberosa Riedel

Theocyrtis tuberosa Riedel, 1959, p. 298, pl. 2, figs. 10, 11. Riedel and Sanfilippo, 1978, p. 78, pl. 1, fig. 11.

Thyrsocyrtis bromia Ehrenberg Thyrsocyrtis bromia Ehrenberg, 1873, p. 260. Riedel and Sanfilippo, 1978, p. 78 , pl. 10, figs. 4,5 .

Thyrsocyrtis tetracantha (Ehrenberg)

Podocyrtis tetracantha Ehrenberg, 1873, p. 254.

Thyrsocyrtis tetracantha (Ehrenberg), Riedel and Sanfilippo, 1978 , p. 80 , pl. 10 , figs. 8,9 .

Thyrsocyrtis triacantha (Ehrenberg)

Podocyrtis triacantha Ehrenberg, 1873, p. 254.

Thyrsocyrtis triacantha (Ehrenberg), Riedel and Sanfilippo, 1978, p. 82 , pl. 10 , figs. 10,11 .

Tristylospyris triceros (Ehrenberg)

Ceratospyris triceros Ehrenberg, 1873, p. 220.

Tristylospyris triceros (Ehrenberg), Riedel and Sanfilippo, 1978, p. 82, pl. 10, fig. 12 .

\section{REFERENCES}

Campbell, A. S., and Clark, B. L., 1944. Miocene radiolarian faunas from southern California. Spec. Pap.-Geol. Soc. Am., no. 51, pp. 1-76. 
Ehrenberg, C. G., 1847. Über die mikroskopischen kieselschaligen Polycystinen als mächtige Gebirgsmasse von Barbados und über das Verhältniss der aus mehr als 300 neuen Arten bestehenden ganz eigenthümlichen Formengruppe jener Felsmasse zu den jetzt lebenden Thieren und zur Kreidebildung. Eine neue Anregung zur Erforschung des Erdlebens. Kgl. Preuss. Akad. Wiss. Berlin, Ber., pp. $40-60$.

1854. Mikrogeologie: Leipzig Voss. xxviii +374 p., Atlas, 31 p., Fortsetzung (1856) 88 p. + 1 p. errata.

, 1860. Über den Tiefgrund des stillen Oceans zwischen Californien und den Sandwich-Inseln aus bis 15600' Tiefe nach Lieut. Brooke. Kgl. Preuss. Akad. Wiss. Berlin, Monatsber., pp. 819-833.

1873. Grössere Felsproben des Polycystinen-Mergels von Barbados mit weiteren Erläuterungen. Kgl. Preus. Akad. Wiss. Berlin, Ber., pp. 213-263.

Foreman, H. P., 1973. Radiolaria of Leg 10 with systematics and ranges for the families Amphipyndacidae, Artostrobiidae, and Theoperidae. In Worzel, J. L., Bryant, W., et al., Init. Repts. DSDP, 10: Washington (U.S. Govt. Printing Office), 407-474.

Haeckel, E., 1887. Report on the Radiolaria collected by $H$. M. S. Challenger during the years 1873-76. Rept. Voy. Challenger. 1873-1876. Zool., 18, Pts. 1, 2:i-clxxxviii, 1-1803.

Harting, P., 1863. Bijdrage tot de kennis der mikroskopische fauna en flora van de Banda Zee. K. Nederl. Akad. Wetensch., Verh., 10:1-34.

Kozlova, G. E., and Gorbovets, A. N., 1966. Radiolyarii verkhnemelovykh i verkhneeotsenovykh otlozhenii Zapadno-Sibirskoi Nizmennosti. Vses. Neft. Nauchno-Issled. Geol.-Razved. Inst. (VNIGRI), Trudy, vypusk 248.

Martini, E., 1971. Standard Tertiary and Quaternary calcareous nannoplankton zonation. In Farinacci, A. (Ed.), Proceedings II Planktonic Conference Roma, pp. 739-785.

Moore, T. C., Jr., 1971. Radiolaria. In Tracey, J. I., Jr., Sutton, G. H., et al., Init. Repts. DSDP, 8: Washington (U.S. Govt. Printing Office), $727-775$.

Nigrini, C. 1974. Cenozoic Radiolaria from the Arabian Sea. DSDP Leg 23. In Davies, T. A., Luyendyk, B. P., et al., Init. Repts. DSDP, 26: Washington (U.S. Govt. Printing Office), 1051-1121.
Okada, H., and Bukry, D., 1980. Supplementary modification and introduction of code numbers to the "Low-latitude coccolith biostratigraphic zonation" (Bukry, 1973; 1975). Mar. Micropaleontol., 5:321-325.

Riedel, W. R., 1953. Mesozoic and Late Tertiary Radiolaria of Rotti. J. Paleontol., 27:805-813.

1957. Radiolaria: a preliminary stratigraphy. Swedish

Deep-Sea Exped. 1947-1948. Repts., 6:59-96.

, 1959. Oligocene and Lower Miocene Radiolaria in tropical Pacific sediments. Micropaleontology, 5:285-302.

Riedel, W. R., and Sanfilippo, A., 1970. Radiolaria, Leg 4, Deep Sea Drilling Project. In Bader, R. G., Gerard, R. D., et al., Init. Repts. DSDP, 4: Washington (U.S. Govt. Printing Office), 503575.

1971. Cenozoic Radiolaria from the western tropical $\mathrm{Pa}$ cific, Leg 7. In Winterer, E. L., Riedel, W. R., et al., Init. Repts. DSDP, 7: Washington (U.S. Govt. Printing Office), 1529-1672.

, 1978. Stratigraphy and evolution of tropical Cenozoic radiolarians. Micropaleontology, 24:61-96.

Sanfilippo, A., and Riedel, W. R., 1970. Post-Eocene "closed" theoperid radiolarians. Micropaleontology, 16:446-462.

1973. Cenozoic Radiolaria (exclusive of theoperids, artostrobiids and amphipyndacids) from the Gulf of Mexico, DSDP Leg 10. In Worzel, J. L., Bryant, W., et al., Init. Repts. DSDP, 10: Washington (U.S. Govt. Printing Office), 475-611.

Theyer, F., Mato, C. Y., and Hammond, S. R., 1978. Paleomagnetic and geochronologic calibration of latest Oligocene to Pliocene radiolarian events, equatorial Pacific. Mar. Micropaleontol., 3: 377-395.

Vinassa de Regny, P. E., 1900. Radiolari Miocenici Italiani. R. Accad. Sci. Ist Bologna Mem., ser. 5, v. 8: 227-257 (565-595).

Westberg, M. J., and Riedel, W. R., 1978. Accuracy of radiolarian correlations in the Pacific Miocene. Micropaleontology, 24:1-23.

Wetzel, O., 1935. Die Mikropalaeontologie des Heiligenhafener Kieseltones (Ober-Eozän). Mit Beiträgen von R. Brandt [Radiolarien] und F. Hustedt [Diatomeen]. Niedersächs. Geol. Ver., Jahresber., 27:41-81. 